\title{
Una luz frente a la pérdida de visión por diabetes
}

\author{
Stéphanie Thébault
}

\section{Resumen}

Encontrar novedosas estrategias terapéuticas para atender el edema macular por diabetes, también llamado edema macular diabético (EMD), es un reto importante en la actualidad ya que esta complicación de la diabetes mellitus de tipo 1 y 2 es la principal causante de pérdida de visión en personas con diabetes. El EMD es una acumulación de líquido en la retina que se debe a un aumento excesivo de la permeabilidad retiniana y la formación de depósitos en la retina central o mácula. La magnitud del problema puede ser apreciada al considerar que 6.4 millones de mexicanos han sido diagnosticados con diabetes y que el 29\% de ellos presenta EMD. En este artículo se revisarán las opciones terapéuticas actuales para el EMD y sus limitantes, y se presentarán las evidencias que apoyan el uso potencial de fármacos bloqueadores de los canales iónicos Transient Receptor Potential Vanilloid 4 (TRPV4) como alternativa terapéutica novedosa para el EMD. Un estudio recién realizado permitió comprobar que el bloqueo de TRPV4 elimina el aumento en la permeabilidad retiniana inducido por diabetes, alteración que promueve el EMD. Por lo anterior y en vista de que antagonistas para TRPV4 se usan actualmente para mitigar el edema pulmonar en pacientes, se espera que próximamente los fármacos bloqueadores de TRPv4 se puedan usar como agentes curativos para el EMD.

Palabras clave: diabetes, edema macular diabético, edema macular por diabetes, canales iónicos Transient Receptor Potential Vanilloid 4 (TRPV4), antagonistas, tratamiento terapéutico.

DOI: http://doi.org/10.22201/codeic.16076079e.2018.v19n5.a3 Recepción: 6/3/2017. Aprobación: 9/8/18. 


\title{
A LIGHT AgAinst the loss OF VISION FROM DIABETES
}

\begin{abstract}
Discovering innovative strategies for treatment of diabetic macular edema (DME) is a real challenge today. DME is a complication of type 1 and type 2 diabetes mellitus and the main cause of vision loss in patients suffering from diabetes. DME is characterized by increased permeability through the blood-retina barrier and hard exudate deposits in the central retina (the macula). The magnitude of the problem can be appreciated in view that 6.4 million of Mexican people have been diagnosed for diabetes, and 29\% of them have DME. Here, we will describe the current treatment options for DME and their limitations, and we will also focus on the potential use of drugs that block Transient Receptor Potential Vanilloid 4 (TRPV4) ion channels. A recent study showed that blockade of TRPV 4 by antagonist drugs abrogates the increased retinal permeability induced by diabetes, which is an alteration that promotes DME. In view that TRPV4 blockers are currently used in clinics to treat pulmonar edema, TRPV4 antagonists are expected to be used as curative agents for DME in the near future.
\end{abstract}

Key words: diabetes, diabetic macular edema, Transient Receptor Potential Vanilloid 4 (TRPV4) ion channels, antagonists, therapeutic treatment.

\section{Stéphanie Thébault}

stephaniethebault@gmail.com

Investigadora en el Instituto de Neurobiología de la Universidad Nacional Autónoma de México (UNAM), Departamento de Neurobiología Celular y Molecular, Laboratorio de Neurobiología Molecular y Celular. Es miembro del Sistema Nacional de Investigadores (SNI), nivel 2 y PRIDE C. Dra. en Ciencias de la Vida y de la Salud por la universidad de Ciencias y Tecnologías de Lille (Francia). Temas de investigación: estudio del papel de canales iónicos y de la actividad eléctrica en la retina y sus implicaciones en diversas patologías retinianas asociadas con inflamación crónica. 


\section{Introducción}

La retina humana es una de las partes más importantes del globo ocular; es la capa sensible que se encuentra en el fondo del ojo, encargada de transformar los impulsos luminosos que recibimos a través de la pupila y la cámara vítrea, en impulsos nerviosos que son analizados por el cerebro para generar imágenes (ver figura 1). En la retina se encuentra la mácula, que tiene cinco milímetros de diámetro y, a su vez, es la parte más importante de la retina ya que en ella reside la visión del detalle (para leer, reconocer caras), y del color.

Figura 1.

Esquema de un ojo y de una sección de retina, que incluye a los vasos sanguíneos intrarretinianos y al epitelio

pigmentario retiniano. El epitelio pigmentario consta de células cúbicas que contienen un pigmento negro, la melanina, y están unidas de manera muy estrecha para formar la parte externa de la barrera hematorretiniana. Se indica donde está la mácula, la fóvea (que es una zona en el centro de la mácula algo deprimida, que presenta un color más oscuro), y la cámara vítrea y la coroides, entre las cuales se encuentra la retina.

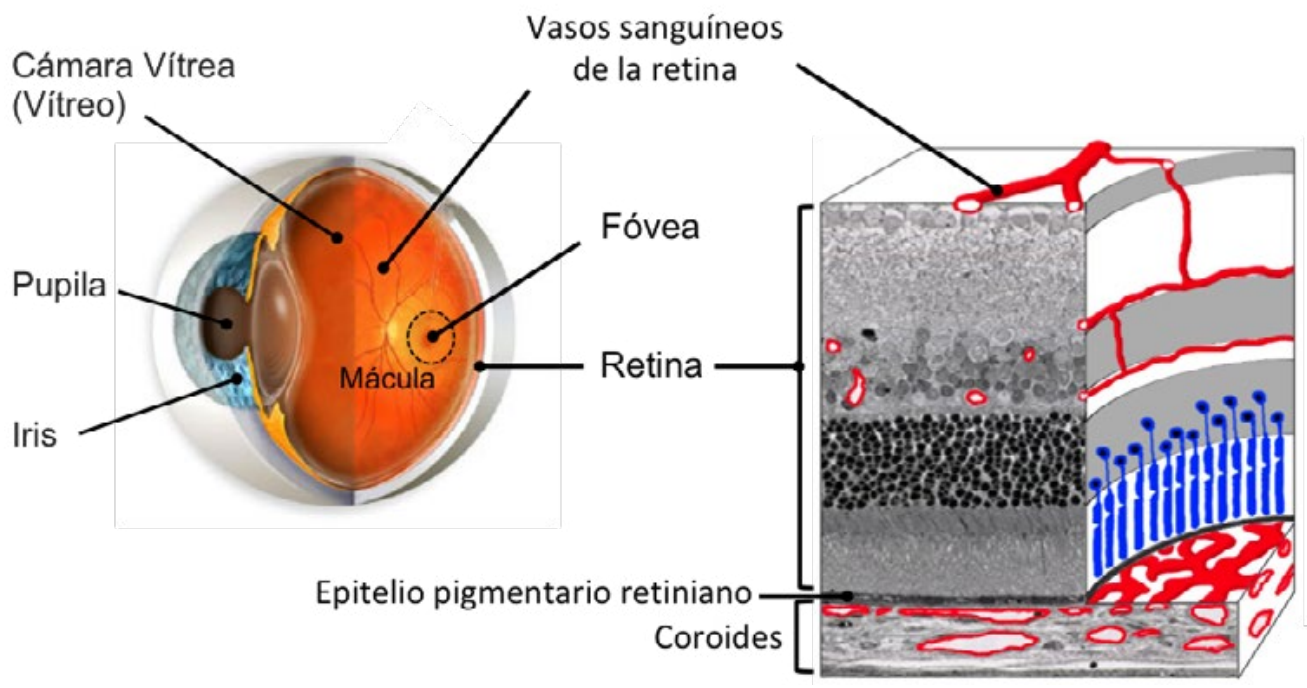

Dentro de las patologías de la retina está el Edema Macular Diabético (EMD). El edema macular es una complicación de la Diabetes Mellitus (DM) (Corcóstegui Guraya y Moreno Manresa, 2001). La Dm puede ser de tipo 1 o tipo 2. La de tipo 1 es una enfermedad autoinmune causada por la destrucción de las células $\beta$ del páncreas, las cuales están encargadas de producir la insulina. Se diagnostica con mayor frecuencia en niños, adolescentes o jóvenes adultos, pero también afecta a personas mayores. En cambio, la Dm tipo 2 es caracterizada por la resistencia a la insulina, una condición en la cual las células no son capaces de utilizar la insulina adecuadamente y/o no hay producción suficiente de insulina. Ya sea de tipo 1 o tipo 2, la Dm se diagnóstica por altos niveles de glucosa en sangre, lo que se denomina hiperglucemia. Si no se trata adecuadamente, la Dm causa daños en vasos sanguíneos grandes y pequeños, como es el caso en el EMD.

El EMD es la acumulación de líquido en la retina, particularmente en la mácula (ver figura 2). Ese líquido proviene de los vasos sanguíneos, cuando la permeabilidad a través de ellos aumenta. Eso puede ocurrir a través de un desbalance entre dos tipos de presiones que existen dentro de los vasos, la presión osmótica y la presión hidrostática. La presión hidrostática es la presión debida al peso del fluido en reposo; aumenta cuando hay hipertensión, por ejemplo, y eso 
Figura 2.

Esquema de un ojo con edema macular por diabetes y de una sección de la mácula, que incluye los vasos sanguíneos intrarretinianos con fuga. lleva a un aumento en la permeabilidad de los vasos. Asimismo, una disminución en la presión osmótica, como pasa cuando los niveles de proteínas en sangre disminuyen, conduce a un aumento en la permeabilidad vascular y favorece la aparición de edema. La permeabilidad vascular en la retina puede también aumentar cuando la permeabilidad a través de la barrera hematorretiniana es alterada. La barrera hematorretiniana se divide en dos componentes, uno interno compuesto por las células vasculares de la retina, y otro externo, formado por las llamadas uniones estrechas que unen las células del epitelio pigmentario de la retina.

Se considera que la hiperglucemia constante causa un aumento en la producción del factor de crecimiento endotelial vascular (VEGF, por sus siglas en inglés) en la retina. El VEGF, además de provocar la formación de nuevos vasos sanguíneos, tiene una importante actividad permeabilizante; es decir, que aumenta la permeabilidad de la barrera hematorretiniana y así ocasiona la salida de líquido del compartimento intravascular (Bhagat et al., 2009; Corcóstegui Guraya y Moreno Manresa, 2001; Thebault, 2011; Zhang et al., 2014). Esto genera un engrosamiento de la retina que puede ser local o que puede afectar áreas más Ojo con edema macular diabético

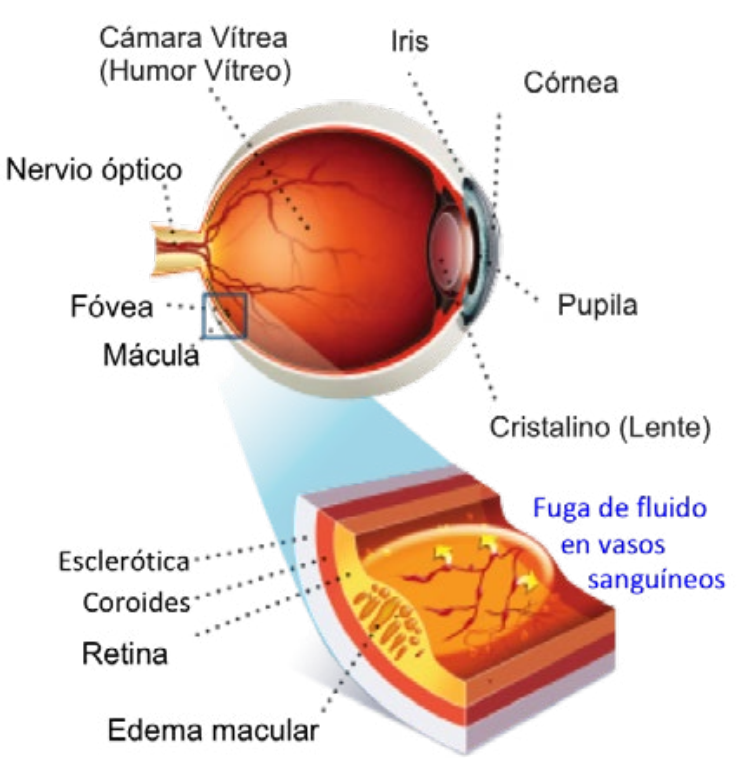
extensas, por lo que el EMD se clasifica en focal y difuso, respectivamente. El EMD, aun cuando no es mortal, se considera un problema de salud pública ya que sus síntomas afectan significativamente la calidad de vida de los pacientes, siendo la principal causa de ceguera en la población laboralmente activa (Yau et al., 2012).

Hasta el 4\% de los pacientes con DM desarrollan problemas que afectan la mácula; hasta el 30\% de los pacientes con edema macular clínicamente significativo perderán capacidad visual de forma moderada. Destaca que el EMD disminuye la agudeza visual en casi la totalidad de los pacientes con DM de tipo 2 (Yau et al., 2012).

Las opciones terapéuticas para el EMD incluyen métodos tradicionales quirúrgicos, a los cuales se han unido en los últimos años nuevas alternativas como la inyección intravítrea de corticoides o de otras sustancias antiangiogénicas, aquellas que inhiben la formación de nuevos vasos sanguíneos (Amoaku, Saker y Stewart, 2015; Hendrick, Gibson y Kulshreshtha, 2015; Regnier et al., 2014; Wang et al., 2015). Dichas terapias previenen la ceguera, lo cual es un logro sustancial; 
sin embargo, tratan los síntomas y no las causas del EMD; son fallidas sin un control riguroso del nivel de azúcar (Corcóstegui Guraya y Moreno Manresa, 2001); además de que pueden conllevar efectos adversos y/o tener efectos benéficos sólo temporales. Por último, no toda la población afectada responde a los tratamientos. Por todo lo anterior, hoy en día, se requieren opciones alternativas para tratar el EMD.

Es en ese contexto que llama la atención un estudio publicado en la revista Scientific Reports (Arredondo Zamarripa et al., 2017), el cual identifica al canal TRPV4 como una nueva diana y por ende, propone una nueva estrategia terapéutica con base en ellos para revertir la fuga vascular retiniana asociada con la diabetes. A continuación, se revisarán los principales hallazgos de este estudio y los razonamientos que permitieron llegar a ellos.

\section{Hacia un nuevo blanco molecular para tratar el EMD}

TRPV4 pertenece a la familia de los canales iónicos, es decir, proteínas que forman un canal o poro en las membranas celulares para dejar pasar iones, partículas con carga eléctrica. El canal TRPV4, permite el paso de iones positivos, Ilamados cationes, como iones de sodio y calcio. En años recientes se ha demostrado la presencia de TRPV4 en la retina (Gilliam y Wensel, 2011). Se ha detectado en las principales células de la barrera hematorretiniana, como son las células de los vasos sanguíneos de la retina (Arredondo Zamarripa et al., 2017; Monaghan et al., 2015) y del epitelio pigmentario retiniano (Zhao et al., 2015; ver figura 3).

Figura 3.

Sección de retina de ratón que incluye a la capa del epitelio pigmentario retiniano y la coroides. Se indica la localización de los canales TRPV4 en diferentes tipos celulares de la retina. EPR. Epitelio Pigmentario Retiniano.

\section{Célula de Müller}

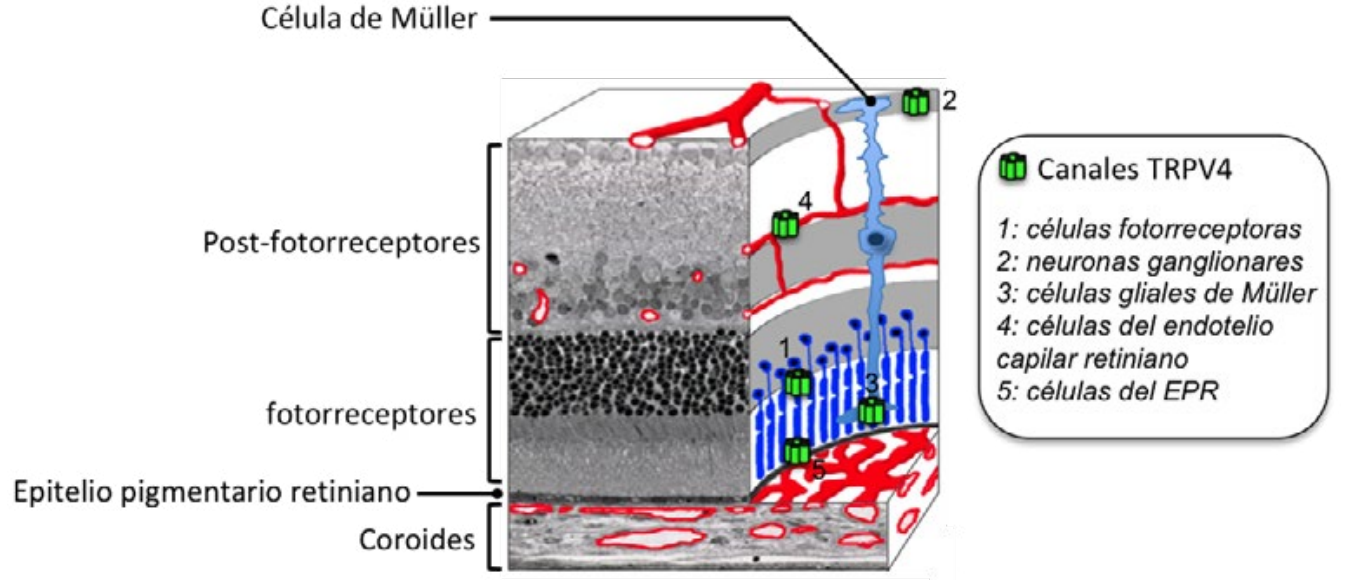

Para poner en contexto la importancia del canal TRPV4 en la fisiología del individuo, hay que recordar que ese canal regula de manera única la permeabilidad de los epitelios (Reiter et al., 2006), los cuales son alfombras de células que fungen como barreras, un ejemplo muy claro es la piel. De manera más específica, TRPV4 regula la permeabilidad a través del endotelio capilar (Cioffi et al., 


\section{. \\ ...es relevante mencionar que la inyección intravítrea es una técnica mínimamente invasiva que tiene como ventajas la máxima biodisponibilidad en el lugar de acción del fármaco...}

2009; Plant y Strotmann, 2007; Wu et al., 2009). El canal se activa por sustancias químicas producidas por los vasos sanguíneos (Watanabe et al., 2002) o por estimulación mecánica asociada al mismo flujo sanguíneo (Liedtke y Friedman, 2003; Liedtke et al., 2003). La activación excesiva de TRPV4 causa la ruptura de la barrera hemato-pulmonar al desprender las células endoteliales de su membrana basal y, por ende, genera edema pulmonar (Hamanaka et al., 2010; Hamanaka et al., 2007; Morty y Kuebler, 2014; Thorneloe et al., 2012; Willette et al., 2008).

En consecuencia, el efecto del bloqueo de TRPV4 por fármacos selectivos (Vincent et al., 2009) ha sido estudiada y se ha mostrado que mitiga el edema y protege la barrera pulmonar (Simonsen et al., 2017; Yin et al., 2008; Yin et al., 2016). Aunado a lo anterior, y de gran relevancia para el uso de antagonistas selectivos de TRPv4 en humano, el uso de dichos fármacos no modifica la frecuencia cardíaca ni la presión arterial (Thorneloe et al., 2012). De hecho, ensayos preclínicos en humanos están actualmente evaluando la respuesta a un antagonista para TRPV4 para tratar la insuficiencia cardíaca estable1, (Grace et al., 2017; Randhawa y Jaggi, 2015), el edema pulmonar² y la enfermedad pulmonar obstructiva crónica³. El bloqueo de TRPV4 inhibe también el edema cerebral en ratones sometidos a isquemia por oclusión arterial (Jie et al., 2015) o trauma cerebral (Lu et al., 2017). Los resultados sobre el efecto inhibitorio de los antagonistas de TRPV4 sobre el edema pulmonar y cerebral representan una ventaja significativa sobre el esquema de tratamiento clínico vigente hasta el momento para tratar estos tipos de edema (esteroides + diuréticos +/- cirugía), ya que los antagonistas de TRPV4 pueden administrarse vía oral en forma aguda o continua, y ejercen efectos antiinflamatorios (Balakrishna et al., 2014; Vergnolle et al., 2010; D. Zhao et al., 2017), antiedema (Balakrishna et al., 2014; Klein et al., 1991) y analgésicos (Thorneloe et al., 2012).

Respecto al tema central de interés de esa revisión, Arredondo Zamarripa y colaboradores comprobaron que fármacos bloqueadores para TRPV4 mitigan los niveles excesivos de permeabilidad retiniana, cuando se administran por vía intravítrea a ratas diabéticas (Arredondo Zamarripa et al., 2017; ver figura 4). Cabe señalar que uno de los inhibidores que probaron, el GSK2193874, deriva del compuesto orgánico heterocíclico quinoleína, y fue descubierto como bloqueador de los canales TRPv4 en el 2017, de manera fortuita. El compuesto, inicialmente diseñado para actuar sobre el sistema cardiovascular, reveló acciones inhibitorias sobre canales iónicos (Cheung et al., 2017). Actualmente se conoce al GSk2193874 como el antagonista más selectivo y potente para TRPV4. Asimismo, ha sido comprobado que este fármaco no inhibe ningún otro canal TRP (Vincent 
Figura 4.

Esquema de un ojo inyectado por vía intravítrea con antagonistas para TRPV4, los cuales han mostrado efectos curativos en contra de la fuga de fluidos a través de la barrera hematorretiniana asociada con la diabetes. et al., 2009). Además, se encontró que TRPV4 por sí mismo no es necesario para regular la función retiniana en condiciones basales, lo que es relevante al momento de proponer el uso de los antagonistas de TRPV4 para tratar el EMD. Adicionalmente, se mostró que el bloqueo de TRPV4 reduce directamente el paso de moléculas entre las células endoteliales que forman los vasos sanguíneos retinianos y también a través del epitelio pigmentario de la retina (Arredondo Zamarripa et al., 2017).

\section{Conclusiones}

Las tres últimas décadas trajeron consigo grandes avances para tratar el edema macular diabético, que es la principal causa de pérdida visual en pacientes diabéticos en México. Sin embargo, las opciones terapéuticas actuales presentan ciertas limitantes que necesitan ser superadas. En este contexto de hallazgos recientes, que han mostrado la capacidad de los antagonistas para los canales iónicos TRPV4 de revertir el aumento en la permeabilidad de la barrera hematorretiniana (alteración que promueve el EMD) en modelos experimentales de diabetes, se ha revelado un potencial terapéutico novedoso de dichos fármacos para tratar el EMD.

Trasladar los resultados de estudios experimentales a la clínica presenta varios retos. Entre ellos, queda por formular una composición farmacéutica antagonista de TRPV4 para ser utilizada para administración intravítrea en el tratamiento del EMD. Al respecto, es relevante mencionar que la inyección intravítrea es una técnica mínimamente invasiva que tiene como ventajas la máxima biodisponibilidad en el lugar de acción del fármaco, la ausencia de efectos secundarios sistémicos, la ausencia de interacciones con otros tratamientos administrados por vía sistémica, mayor calidad de vida al ser un tratamiento ambulatorio y no requerir hospitalización ni administración diaria de goteros, y a un precio accesible, sin mencionar el ahorro directo al sector salud. Por todo lo anterior, se puede considerar que existen muchos elementos a favor del uso de los antagonistas para TRPV4 como una alternativa terapéutica en el tratamiento del EMD en un futuro próximo. 


\section{Referencias}

- Amoaku, W. M., Saker, S. y Stewart, E. A. (2015). A review of therapies for diabetic macular oedema and rationale for combination therapy. Eye (Lond), 29(9), 11151130. Dol: https://doi.org/10.1038/eye.2015.110.

- Arredondo Zamarripa, D., Noguez Imm, R., Bautista Cortes, A. M., Vazquez Ruiz, O., Bernardini, M., Fiorio Pla, A., ... Thebault, S. (2017). Dual contribution of TRPv4 antagonism in the regulatory effect of vasoinhibins on blood-retinal barrier permeability: diabetic milieu makes a difference. Sci Rep, 7(1), 13094. Dol: https://doi. org/10.1038/s41598-017-13621-8.

* Balakrishna, S., Song, W., Achanta, S., Doran, S. F., Liu, B., Kaelberer, M. M., ... Jordt, S. E. (2014). TRPV4 inhibition counteracts edema and inflammation and improves pulmonary function and oxygen saturation in chemically induced acute lung injury. Am J Physiol Lung Cell Mol Physiol, 307(2), L158-172. Dol: https://doi.org/10.1152/ ajplung.00065.2014.

* Bhagat, N., Grigorian, R. A., Tutela, A. y Zarbin, M. A. (2009). Diabetic macular edema: pathogenesis and treatment. Surv Ophthalmol, 54(1), 1-32. Dol: https://doi. org/10.1016/j.survophthal.2008.10.001.

- Cheung, M., Bao, W., Behm, D. J., Brooks, C. A., Bury, M. J., Dowdell, S. E., ... Thorneloe, K. S. (2017). Discovery of GSK2193874: An Orally Active, Potent, and Selective Blocker of Transient Receptor Potential Vanilloid 4. ACS Med Chem Lett, 8(5), 549554. Dol: https://doi.org/10.1021/acsmedchemlett.7b00094.

Cioffi, D. L., Lowe, K., Alvarez, D. F., Barry, C. y Stevens, T. (2009). TRPing on the lung endothelium: calcium channels that regulate barrier function. Antioxid Redox Signal, 11(4), 765-776. Dol: https://doi.org/10.1089/ARS.2008.2221.

* Corcóstegui Guraya, B. yMoreno Manresa, J. (2001). Capítulo 3. Edema macular diabético. Mesa Redonda, 77 Congreso de la Sociedad Española de Oftalmología, Barcelona, 2001, 37-51.

* Gilliam, J. C.y Wensel, T. G. (2011). TRP channel gene expression in the mouse retina. Vision Res, 51(23-24), 2440-2452. Dol: https://doi.org/10.1016/j.visres.2011.10.009.

* Grace, M. S., Bonvini, S. J., Belvisi, M. G. y McIntyre, P. (2017). Modulation of the TRPV4 ion channel as a therapeutic target for disease. Pharmacol Ther. Dol: https:// doi.org/10.1016/j.pharmthera.2017.02.019.

- Hamanaka, K., Jian, M. Y., Townsley, M. I., King, J. A., Liedtke, W., Weber, D. S., . . Parker, J. C. (2010). TRPV4 channels augment macrophage activation and ventilatorinduced lung injury. Am J Physiol Lung Cell Mol Physiol, 299(3), L353-362. Dol: https:// doi.org/10.1152/ajplung.00315.2009.

- Hamanaka, K., Jian, M. Y., Weber, D. S., Alvarez, D. F., Townsley, M. I., Al-Mehdi, A. B., ... Parker, J. C. (2007). TRPV4 initiates the acute calcium-dependent permeability increase during ventilator-induced lung injury in isolated mouse lungs. Am J Physiol Lung Cell Mol Physiol, 293(4), L923-932. Dol: https://doi.org/10.1152/ ajplung.00221.2007. 
* Hendrick, A. M., Gibson, M. V. y Kulshreshtha, A. (2015). Diabetic Retinopathy. Prim Care, 42(3), 451-464. Dol: https://doi.org/10.1016/j.pop.2015.05.005.

* Jie, P., Tian, Y., Hong, Z., Li, L., Zhou, L., Chen, L. y Chen, L. (2015). Blockage of transient receptor potential vanilloid 4 inhibits brain edema in middle cerebral artery occlusion mice. Front Cell Neurosci, 9, 141. Dol: https://doi.org/10.3389/fncel.2015.00141

* Klein, B. E., Moss, S. E., Klein, R. y Surawicz, T. S. (1991). The Wisconsin Epidemiologic Study of Diabetic Retinopathy. XIII. Relationship of serum cholesterol to retinopathy and hard exudate. Ophthalmology, 98(8), 1261-1265. Recuperado de: https://www.ncbi.nlm.nih.gov/pubmed/1923364.

- Liedtke, W. y Friedman, J. M. (2003). Abnormal osmotic regulation in TRPV4-/mice. Proc Natl Acad Sci U S A, 100(23), 13698-13703. Dol: https://doi.org/10.1073/ pnas.1735416100.

- Liedtke, W., Tobin, D. M., Bargmann, C. I. y Friedman, J. M. (2003). Mammalian TRPV4 (VR-OAC) directs behavioral responses to osmotic and mechanical stimuli in Caenorhabditis elegans. Proc Natl Acad Sci U S A, 100 Suppl 2, 14531-14536. Dol: https://doi.org/10.1073/pnas.2235619100.

- Lu, K. T., Huang, T. C., Tsai, Y. H. y Yang, Y. L. (2017). Transient receptor potential vanilloid type 4 channels mediate $\mathrm{Na}-\mathrm{K}$-Cl-co-transporter-induced brain edema after traumatic brain injury. J Neurochem, 140(5), 718-727. Dol: https://doi. org/10.1111/jnc.13920.

Monaghan, K., McNaughten, J., McGahon, M. K., Kelly, C., Kyle, D., Yong, P. H., . . Curtis, T. M. (2015). Hyperglycemia and Diabetes Downregulate the Functional Expression of TRPV4 Channels in Retinal Microvascular Endothelium. PLoS One, 10(6), e0128359. Dol: https://doi.org/10.1371/journal.pone.0128359.

Morty, R. E. y Kuebler, W. M. (2014). TRPV4: an exciting new target to promote alveolocapillary barrier function. Am J Physiol Lung Cell Mol Physiol, 307(11), L817-821. Dol: https://doi.org/10.1152/ajplung.00254.2014.

* Plant, T. D. y Strotmann, R. (2007). Trpv4. Handb Exp Pharmacol. (179), 189-205. DoI: https://doi.org/10.1007/978-3-540-34891-7_11.

* Randhawa, P. K. y Jaggi, A. S. (2015). TRPV4 channels: physiological and pathological role in cardiovascular system. Basic Res Cardiol, 110(6), 54. Dol: https://doi. org/10.1007/s00395-015-0512-7.

* Regnier, S., Malcolm, W., Allen, F., Wright, J. y Bezlyak, V. (2014). Efficacy of anti-VEGF and laser photocoagulation in the treatment of visual impairment due to diabetic macular edema: a systematic review and network meta-analysis. PLoS One, 9(7), e102309. Dol: https://doi.org/10.1371/journal. pone.0102309.

* Reiter, B., Kraft, R., Gunzel, D., Zeissig, S., Schulzke, J. D., Fromm, M. y Harteneck, C. (2006). TRPV4-mediated regulation of epithelial permeability. FASEB J, 20(11), 1802-1812. Dol: https://doi.org/10.1096/fj.06-5772com. 
* Simonsen, U., Wandall-Frostholm, C., Olivan-Viguera, A. y Kohler, R. (2017). Emerging roles of calcium-activated K channels and TRPV4 channels in lung oedema and pulmonary circulatory collapse. Acta Physiol (Oxf), 219(1), 176-187. Dol: https://doi. org/10.1111/apha.12768.

- Thebault, S. (2011). El epitelio pigmentario retiniano como componente de la barrera hemato-retiniana: implicación en la retinopatía diabética. Revista Digital Universitaria, 12(3). Recuperado de: http://www.revista.unam.mx/vol.12/num3/art31/index.html.

* Thorneloe, K. S., Cheung, M., Bao, W., Alsaid, H., Lenhard, S., Jian, M. Y., ... Willette, R. N. (2012). An orally active TRPV4 channel blocker prevents and resolves pulmonary edema induced by heart failure. Sci Trans/ Med, 4(159), 159ra148. Dol: https:// doi.org/10.1126/scitranslmed.3004276.

* Vergnolle, N., Cenac, N., Altier, C., Cellars, L., Chapman, K., Zamponi, G. W., . . . Bunnett, N. W. (2010). A role for transient receptor potential vanilloid 4 in tonicityinduced neurogenic inflammation. Br J Pharmacol, 159(5), 1161-1173. Dol: https:// doi.org/10.1111/j.1476-5381.2009.00590.x.

* Vincent, F., Acevedo, A., Nguyen, M. T., Dourado, M., DeFalco, J., Gustafson, A., . . . Duncton, M. A. (2009). Identification and characterization of novel TRPV4 modulators. Biochem Biophys Res Commun, 389(3), 490-494. Dol: https://doi.org/10.1016/j. bbrc.2009.09.007.

* Wang, J. K., Huang, T. L., Su, P. Y. y Chang, P. Y. (2015). An updated review of longterm outcomes from randomized controlled trials in approved pharmaceuticals for diabetic macular edema. Eye Sci, 30(4), 176-183. Recuperado de: http://ykxb. amegroups.com/article/view/3523/4246.

-Watanabe, H., Davis, J. B., Smart, D., Jerman, J. C., Smith, G. D., Hayes, P., ... Nilius, B. (2002). Activation of TRPV4 channels (hVRL-2/mTRP12) by phorbol derivatives. J Biol Chem, 277(16), 13569-13577. Dol: https://doi.org/10.1074/jbc.M200062200.

* Willette, R. N., Bao, W., Nerurkar, S., Yue, T. L., Doe, C. P., Stankus, G., . . Xu, X. (2008). Systemic activation of the transient receptor potential vanilloid subtype 4 channel causes endothelial failure and circulatory collapse: Part 2. J Pharmacol Exp Ther, 326(2), 443-452. Dol: https://doi.org/10.1124/jpet.107.134551.

- Wu, S., Jian, M. Y., Xu, Y. C., Zhou, C., Al-Mehdi, A. B., Liedtke, W., ... Townsley, M. I. (2009). Ca2+ entry via alpha1G and TRPV4 channels differentially regulates surface expression of P-selectin and barrier integrity in pulmonary capillary endothelium. Am J Physiol Lung Cell Mol Physiol, 297(4), L650-657. Dol: https://doi.org/10.1152/ ajplung.00015.2009.

- Yau, J. W., Rogers, S. L., Kawasaki, R., Lamoureux, E. L., Kowalski, J. W., Bek, T., . . . Meta-Analysis for Eye Disease Study, G. (2012). Global prevalence and major risk factors of diabetic retinopathy. Diabetes Care, 35(3), 556-564. Dol: https://doi. org/10.2337/dc11-1909.

* Yin, J., Hoffmann, J., Kaestle, S. M., Neye, N., Wang, L., Baeurle, J., ... Kuebler, W. M. (2008). Negative-feedback loop attenuates hydrostatic lung edema via a cGMPdependent regulation of transient receptor potential vanilloid 4. Circ Res, 102(8), 966-974. Dol: https://doi.org/10.1161/CIRCRESAHA.107.168724. 
Yin, J., Michalick, L., Tang, C., Tabuchi, A., Goldenberg, N., Dan, Q., ... Kuebler, W. M. (2016). Role of Transient Receptor Potential Vanilloid 4 in Neutrophil Activation and Acute Lung Injury. Am J Respir Cell Mol Biol, 54(3), 370-383. Dol: https://doi. org/10.1165/rcmb.2014-02250C.

* Zhang, X., Zeng, H., Bao, S., Wang, N., \& Gillies, M. C. (2014). Diabetic macular edema: new concepts in patho-physiology and treatment. Cell Biosci, 4, 27. Dol: https:// doi.org/10.1186/2045-3701-4-27.

Z Zhao, D., Nguyen, C. T., Wong, V. H., Lim, J. K., He, Z., Jobling, A. I., .. Bui, B. V. (2017). Characterization of the Circumlimbal Suture Model of Chronic IOP Elevation in Mice and Assessment of Changes in Gene Expression of Stretch Sensitive Channels. Front Neurosci, 11, 41. Dol: https://doi.org/10.3389/fnins.2017.00041.

* Zhao, P. Y., Gan, G., Peng, S., Wang, S. B., Chen, B., Adelman, R. A., \& Rizzolo, L. J. (2015). TRP Channels Localize to Subdomains of the Apical Plasma Membrane in Human Fetal Retinal Pigment Epithelium. Invest Ophthalmol Vis Sci, 56(3), 19161923. Dol: https://doi.org/10.1167/iovs.14-15738.

\section{Cómo citar este artículo}

* Thébault, Stéphanie (2018). Una luz frente a la pérdida de visión por diabetes. Revista Digital Universitaria (RDU). Vol. 19, núm. 5 septiembre-octubre. DOI: http:// doi.org/10.22201/codeic.16076079e.2018.v19n5.a3. 